\title{
Vibration control of a linear flexible beam structure excited by multiple harmonics
}

\author{
Bassam A. Albassam \\ Mechanical Engineering Department, King Saud University, Riyadh, 11421, Saudi Arabia \\ Corresponding Author : Albassam@ksu.edu.sa
}

$\begin{array}{ll}\text { Submitted } & : 20 / 10 / 2019 \\ \text { Revised } & : 03 / 01 / 2020 \\ \text { Accepted } & : 02 / 02 / 2020\end{array}$

\begin{abstract}
This paper deals with designing a control force to create nodal point(s) having zero displacement and/or zero slope at selected locations in a vibrating beam structure excited by multiple harmonic forces. It is shown that the steady state vibrations at desired points can be eliminated using applied control forces. The control forces design method is implemented using dynamic Green's functions that transform the equations of motion from differential to algebraic equations, in which the resulting solution is analytic and exact. The control problem is greatly simplified by utilizing the superposition principle that leads to calculating the control forces to create node(s) for each excitation frequency independently. The calculated control forces can be realized using passive elements such as masses and springs connected to the beam having reaction forces equal to the calculated control forces. The effectiveness of the proposed method is demonstrated on various cases using numerical examples. Through examples, it was shown that creating node(s) with zero deflection, as well as zero slope, not only results in isolated stationary points, but also suppresses the vibrations along a wide region of the beam.
\end{abstract}

Keywords: Vibration control; Green's function; Flexible arm; Vibration absorber.

\section{INTRODUCTION}

In recent years, there has been a considerable interest in modeling and control of flexible structures. This is due to the use of lightweight materials for the purposes of speed and fuel efficiency. Therefore, these structures suffer from persistent vibrations due to low internal damping, causing problems such as human discomfort, component failure, performance degradation, noise, and many other problems. Moreover, the performance is substantially degraded in some structures equipped with sensitive elements due to the occurrence of vibration. Therefore, it is desired to suppress the vibration from specific part of the structure more than other parts. For example, large flexible space structures are usually built from lightweight materials having low damping. Therefore, if these structures are exposed to any excitation, then the vibrations may propagate throughout the whole structure. In such a case, it is desirable to eliminate the vibrations in some sensitive parts, where an extremely sensitive antenna is installed.

There are a number of ways to eliminate or minimize the vibrations in a flexible structure. The first one is to remove the disturbances that excite the structure. Unfortunately, it may be difficult or impossible to interfere with the nature of the driving force. The other method is to adjust the structural system to change its dynamics that results in less vibrations, especially during resonance. This is, sometimes, a difficult approach to be applied on existing 
structures. The third solution is to utilize one of the vibration control techniques to either absorb the vibrational energy or load the transmission path of the disturbing vibration.

Many researchers have investigated the topic of vibration control using the abovementioned methods. The first vibration absorber has been designed and patented by Frahm (1911). Ormondroyd and Den Hartog (1928) have described the first mathematical theory for the design of the dynamic vibration absorber (DVA). Korenev and Reznikov (1993) have presented theoretical and practical studies on the design of dynamic vibration absorbers.

The problem of beam vibrations with attached passive components, for example, masses and springs, falls in the class of the dynamics of combined systems. The free vibrations of such systems are studied extensively in the literature (Cha 2001; Low 2003; Banerjee and Sobey 2003; Wu and Chou 1999). The authors of these papers have focused their research on evaluating the free vibrations by calculating the natural frequencies and mode shapes. Other researchers have used the assumed modes method to solve different vibration problems in structures (for example, Ginsberg 2001). Cha and Pierre (1999) have used the assumed modes method to create points with zero displacements to the normal modes of a beam by attaching masses and springs and properly selecting the values of their parameters. Cha and Zhou (2006) have enforced points with both zero displacements and zero slopes in a linear structure using elastically attached masses and rotational springs during harmonic excitations. The dynamic green function has been utilized by Alsaif and Foda (2002) to compute the optimal values of masses and/or springs and their locations along a beam in order to reduce the vibrations in a specified region. Similarly, Foda and Albassam (2006) have derived exact solutions for the steady state response of a harmonically excited Timoshenko beam using Green's function by attaching springs and/or masses. Their objective was to confine the vibrations in a region of the beam. Foda and Alsaif (2009) have developed a numerical method to impose nodes with zero deflection, as well as slope, on a beam structure excited by harmonic force using translational and rotational oscillators.

On the other hand, points or regions with zero deflections can be created in distributed parameter systems using active vibration control methods. Ram (2002) and Singh and Ram (2003) have obtained closed form expression for the feedback control gain to impose a node at the end of axially vibrating rods and transversely vibrating beams using displacement feedback control. Albassam (2019) has obtained analytical expressions for the gains of the feedback control forces in order to create nodes with zero displacements and zero slopes using dynamic Green's function.

It is to be noted that all of the above researchers have considered the problem of forcing input with single frequency. Unfortunately, in real applications, the structure, generally, is exposed to excitations with multiple frequencies. Cha (2005) has utilized spring and mass elements to induce nodes along harmonically excited beam with an added constraint on the vibration amplitude of oscillator mass. Later, Cha and Ren (2006) have generalized the method to induce nodes for beams excited by multiple frequencies using the assumed modes method. Subsequently, and due to the difficulty of the numerical procedure reported in Cha and Ren (2006), Cha and Buyco (2015) have developed an efficient method to tune the oscillator parameters in order to induce nodes on a beam using the active force approach.

In the present work, control forces are designed for the purpose of creating nodes (points with zero displacements) or fixed nodes (points with zero displacements and zero slopes) along any beam structure during harmonic excitation using dynamic Green's function. It is assumed that the beam follows the Euler-Bernoulli beam theory assumptions. In addition, the effects of internal and external damping are included in the beam model. The problem is formulated using dynamic Green's function, which results in exact and straightforward solution. This method is free from numerical inaccuracies when compared with other approximate methods that utilize modal superposition techniques. Furthermore, the boundary conditions are included into the derivation of the Green's function of the corresponding beam. 
The main contribution of the paper, compared with similar research work, is the utilization of dynamic Green's function that results in an algebraic equation for the steady-state beam deflection. Furthermore, since beam boundary conditions are embedded in the derivation of Green's function, different beam types can be considered. Therefore, the resulting solution is simple, exact, and general.

The paper is organized as follows. Following this introduction, the equation of motion in the form of partial differential equation is derived in Section 2 using Hamilton's principle. In Section 3, the steady-state solution for the beam deflection is obtained using Green's function. The numerical procedure to impose multiple nodes and fixed nodes is presented in Section 4. Section 5 demonstrates the application of passive elements, such as masses and springs, as means to generate the applied control forces. The application of the proposed vibration control technique on numerical examples for simulations and discussions is shown in Section 6. Finally, conclusions and summary of the paper findings are laid out in Section 7.

\section{EQUATION OF MOTION}

The transverse vibration problem of a uniform Euler Bernoulli beam of length $L$, originally at rest, is shown in Figure 1 . The beam can have any boundary conditions at its two ends. It is assumed that the beam vibration is caused by external harmonic forces, represented by $f(x, t)$, having multiple frequencies, given by

$$
f(x, t)=\sum_{i=1}^{p} F_{i} e^{j \omega_{i} t} \delta\left(x-x_{f_{i}}\right)
$$

where $p$ is the number of excitation forces, $F_{i}, \omega_{i}$, and $x_{f_{i}}$ are the amplitude, frequency, and location of the $i^{\text {th }}$ excitation force, respectively, $j=\sqrt{-1}$ is the imaginary unit, and $\delta()$ is the Dirac Delta function.

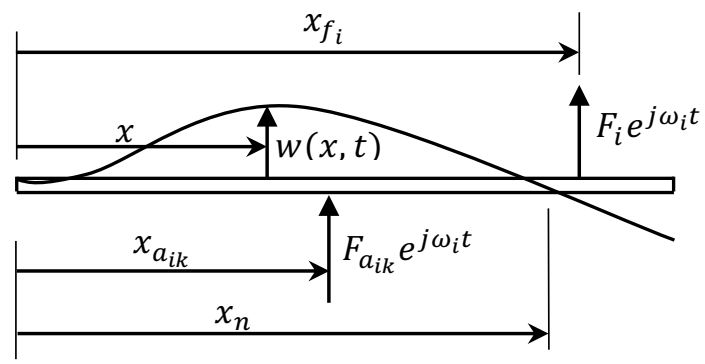

Figure 1. Configuration of a harmonically excited elastic beam with vibration control force.

It is assumed that a certain application requires to impose $r$ nodes on the beam structure using the applied control forces

given by

$$
f_{a}(x, t)=\sum_{i=1}^{p} \sum_{l=1}^{r} F_{a_{i l}} e^{j \omega_{i} t} \delta\left(x-x_{a_{i l}}\right)
$$

where $F_{a_{i l}}$ and $x_{a_{i l}}$ are the amplitude and location of the $i l^{\text {th }}$ applied force. In general, a total of $p \times r$ applied forces are required to impose $r$ nodes on a beam structure that is excited by $p$ harmonic forces (Cha and Ren 2006 and Cha and

Buyco 2015). 
The first step in designing the applied control forces to impose the desired nodes on the beam structure is to derive the equation of motion for the beam system shown in Figure 1 using Hamilton's principle. The kinetic energy of the beam system can be

written as

$$
T=\frac{1}{2} \int_{0}^{L} \rho A\left(\frac{\partial w(x, t)}{\partial t}\right)^{2} d x
$$

where $\rho$ is the beam material density in $\mathrm{kg} / \mathrm{m}^{3}, A$ is the beam cross sectional area in $\mathrm{m}^{2}, w(x, t)$ is the beam transverse deflection in $m$, and $t$ is the time in $s$. The potential energy can be written as:

$$
V=\frac{1}{2} \int_{0}^{L} E I\left(\frac{\partial^{2} w(x, t)}{\partial x^{2}}\right)^{2} d x
$$

where $E$ is the beam modulus of elasticity, in $N / \mathrm{m}^{2}$, and $I$ is the moment of inertia of the beam cross sectional area, in $m^{4}$. The virtual work done by the external nonconservative forces can be expressed as

$$
W_{n c}=\int_{0}^{L}\left[f(x, t)+f_{a}(x, t)+f_{d}(x, t)\right] w(x, t) d x
$$

where $f_{d}(x, t)$ represents the damping forces, given by

$$
f_{d}(x, t)=c_{1} \frac{\partial w(x, t)}{\partial t}+c_{2} \frac{\partial^{5} w(x, t)}{\partial x^{4} \partial t}
$$

where $c_{1}$ is the coefficient of external viscous damping due to friction with the surroundings, in N.s $/ \mathrm{m}$, and $c_{2}$ is the coefficient of internal viscoelastic damping, in N.s.m ${ }^{3}$.

The equation of motion can be derived by applying Hamilton's principle, given by

$$
\int_{t_{1}}^{t_{2}} \delta(T-V) d t+\int_{t_{1}}^{t_{2}} \delta W_{n c} d t=0
$$

after substituting Equations (3), (4), (5), and (6) and performing the variational mathematics with integration by parts, the following equation of motion can be obtained.

$$
E I \frac{\partial^{4} w(x, t)}{\partial x^{4}}+\rho A \frac{\partial^{2} w(x, t)}{\partial t^{2}}+c_{1} \frac{\partial w(x, t)}{\partial t}+c_{2} \frac{\partial^{5} w(x, t)}{\partial x^{4} \partial t}=\sum_{i=1}^{p} F_{i} e^{j \omega_{i} t} \delta\left(x-x_{f_{i}}\right)+\sum_{i=1}^{p} \sum_{l=1}^{r} F_{a_{i l}} e^{j \omega_{i} t} \delta\left(x-x_{a_{i l}}\right)
$$

It is to be noted that, since damping exists, complex exponential functions are used in the representation of the excitation and applied control forces resulting in simpler mathematical manipulations. Consequently, it assumed that the input forces are given by their real parts which results in the real part of the beam deflection. 


\section{PROBLEM FORMULATION}

In this section, the steady-state beam deflection, in the form of algebraic equation, is solved using Green's function. Because the system under consideration is assumed to be linear, the principle of superposition applies and the solution of Equation (8) is assumed to have the following form:

$$
w(x, t)=\sum_{i=1}^{p} W_{i}(x) e^{j \omega_{i} t}
$$

where $W_{i}(x)$ is the steady-state deflection of the beam at location $x$ due to harmonic excitation and control forces. Consequently, the system will execute a simple harmonic motion with the same response frequency as the driving frequency.

Substituting Equation (9) into Equation (8) results in

$$
\sum_{i=1}^{p}\left[\left(E I+j \omega_{i} c_{2}\right) \frac{d^{4} W_{i}(x)}{d x^{4}}+\left(j \omega_{i} c_{1}-\rho A \omega_{i}^{2}\right) W_{i}(x)\right] e^{j \omega_{i} t}=\sum_{i=1}^{p}\left[F_{i} \delta\left(x-x_{i f}\right)+\sum_{l=1}^{r} F_{a_{i l}} \delta\left(x-x_{a_{i l}}\right)\right] e^{j \omega_{i} t}(10)
$$

Once again, and by virtue of the superposition principle, Equation (10) reveals that the governing equation for the steady-state response due to each harmonic excitation force can be considered separately as

$$
\begin{gathered}
\left(E I+j \omega_{i} c_{2}\right) \frac{d^{4} W_{i}(x)}{d x^{4}}+\left(j \omega_{i} c_{1}-\rho A \omega_{i}^{2}\right) W_{i}(x)=F_{i} \delta\left(x-x_{i f}\right)+\sum_{l=1}^{r} F_{a_{i l}} \delta\left(x-x_{a_{i l}}\right) \\
i=1,2, \ldots, p
\end{gathered}
$$

Therefore, the design of the applied control force(s) and the resulting steady-state response can be calculated separately for each excitation force and then combined, using Equation (9), to obtain the total steady-state response due to the total number of distinct harmonic excitations.

At this stage, it is convenient to work with dimensionless quantities so that the analysis becomes more general. Therefore, we define the following dimensionless variables and coefficients:

$$
\hat{x}=\frac{x}{L}, \quad \widehat{W}_{i}=\frac{W_{i}}{W_{0_{i}}}, \quad \widehat{\omega}_{i}=\frac{\omega_{i}}{\omega_{0}}
$$

where

$$
W_{0_{i}}=\frac{F_{i} L^{3}}{E I} \quad, \quad \omega_{0}=\sqrt{\frac{E I}{\rho A L^{4}}}
$$

and

$$
\hat{c}_{1}=\frac{\omega_{0} L^{4} c_{1}}{E I}, \quad \hat{c}_{2}=\frac{\omega_{0} c_{2}}{E I}, \quad \hat{F}_{a_{i l}}=\frac{F_{a_{i l}}}{F_{i}},
$$

The resulting dimensionless form of Equation (11) becomes 


$$
\widehat{W}_{i}^{4}(\hat{x})-\frac{\widehat{\omega}_{i}^{2}-j \widehat{\omega}_{i} \hat{c}_{1}}{1+j \widehat{\omega}_{i} \hat{c}_{2}} \widehat{W}_{i}(\hat{x})=\frac{1}{1+j \widehat{\omega}_{i} \hat{c}_{2}}\left[\hat{\delta}\left(\hat{x}-\hat{x}_{i f}\right)+\sum_{l=1}^{r} \hat{F}_{a_{i l}} \hat{\delta}\left(\hat{x}-\hat{x}_{a_{i l}}\right)\right], \quad i=1,2, \ldots, p
$$

where $\widehat{W}_{i}^{(4)}(\hat{x})=\frac{d^{4} \widehat{W}}{d \hat{x}^{4}}$ and $\hat{\delta}(L \hat{x})=\frac{1}{L} \hat{\delta}(\hat{x})$

Before proceeding with the solution of Equation (15), the caret on the dimensionless quantities is dropped for the sake of convenience. The solution of Equation (15) is sought by utilizing the dynamic Green's function. Hence, if the dynamic Green's function, denoted by $G_{i}(x, u)$, is known then the solution of Equation (15) is given by the following integral form:

$$
W_{i}(x)=\frac{1}{1+j \omega_{i} c_{2}} \int_{0}^{1}\left[G_{i}(x, u) \delta\left(u-x_{i f}\right)+\sum_{l=1}^{r} F_{a_{i l}} G_{i}(x, u) \delta\left(u-x_{a_{i l}}\right)\right] d u
$$

The Green's function, $G_{i}(x, u)$, for the beam is defined as the response at position $x$ due to a unit concentrated force applied at position $u$. Utilizing the properties of the Dirac Delta function, the integration in Equation (16) can be carried out to give

$$
W_{i}(x)=\frac{1}{1+j \omega_{i} c_{2}}\left[G_{i}\left(x, x_{i f}\right)+\sum_{l=1}^{r} F_{a_{i l}} G_{i}\left(x, x_{a_{i l}}\right)\right]
$$

Equation (17) is an algebraic equation that provides the exact closed form solution for the beam steady-state deflection at any location resulting from the application of the excitation and control forces. It requires the calculations of the Green's function, $G_{i}(x, u)$, that can be obtained from the solution of the following differential equation (Roach 1970):

$$
\frac{d^{4} G_{i}(x, u)}{d x^{4}}-q_{i}^{4} G_{i}(x, u)=\delta(x-u)
$$

where $q_{i}^{4}$ is given by

$$
q_{i}^{4}=\frac{\omega_{i}^{2}-j \omega_{i} c_{1}}{1+j \omega_{i} c_{2}}
$$

From Equations (18) and (19), it is observed that Green's function varies with the excitation frequency among other parameters. Furthermore, Green's function becomes complex if either the beam external damping or internal damping is present. Several researchers have reported the solution of Equation (18) (see, for example, Roach 1970 and Roach 1982) for different beam boundary conditions. For example, Green's function for a simply supported beam is given by

$$
G_{i}(x, u)=\frac{1}{2 q_{i}^{3} \sin \left(q_{i} L\right) \sinh \left(q_{i} L\right)} \begin{cases}g_{i}(x, u), & 0 \leq x \leq u \\ g_{i}(u, x), & x \leq u \leq L\end{cases}
$$


where

$$
g_{i}(x, u)=\sinh \left(q_{i} L\right) \sin \left(q_{i} x\right) \sin \left(q_{i} L-q_{i} u\right)-\sin \left(q_{i} L\right) \sinh \left(q_{i} x\right) \sinh \left(q_{i} L-q_{i} u\right)
$$

and $g_{i}(u, x)$ is obtained by replacing $x$ with $u$ in Equation (21).

\section{ENFORCING NODE(S) AND FIXED NODE(S)}

\section{One Node}

The derivations for the analytical solution of the applied control force that results in creating a point, at location $x_{n}$, on a beam having zero deflection, called a node, is presented in this section. First, the deflection at location $x_{n}$ is defined using Equation (17) to obtain

$$
W_{i}\left(x_{n}\right)=\frac{1}{1+j \omega_{i} c_{2}}\left[G_{i}\left(x_{n}, x_{i f}\right)+F_{a_{i 1}} G_{i}\left(x_{n}, x_{a_{i 1}}\right)\right]
$$

To create a stationary point at location $x_{n}$ on the beam, we set the righthand side of Equation (22) to zero, to give

$$
G_{i}\left(x_{n}, x_{i f}\right)+F_{a_{i 1}} G_{i}\left(x_{n}, x_{a_{i 1}}\right)=0
$$

Solving Equation (23) for the amplitude of the control force results in

$$
F_{a_{i 1}}=-\frac{G_{i}\left(x_{n}, x_{i f}\right)}{G_{i}\left(x_{n}, x_{a_{i 1}}\right)}
$$

As seen in Equation (24), the calculation of the amplitude of the $i 1^{\text {th }}$ applied control force to create a stationary point (or node) on the beam that is vibrating as a result of the application of the excitation force having amplitude $F_{i}$ and frequency $\omega_{i}$ is very simple, exact, and straightforward. Next, the calculations for the amplitudes of the other excitation forces to create a stationary point on the same location are performed separately. Finally, the steady-state deflection $W_{i}(x)$ can be calculated using Equation (17) and the total response is obtained using Equation (9).

\section{Multiple Nodes}

In this section, we outline the numerical procedure for calculating the amplitudes of the applied control forces with the objective of creating $r$ nodes on the beam. In general, each node requires one control force to be designed and applied to the beam. Substituting $x=x_{n_{1}}, x_{n_{2}}, \ldots, x_{n_{r}}$ in Equation (17) results in $r$ simultaneous linear algebraic equations in $r$ unknowns. The unknowns are the steady-state amplitudes for the applied control forces, i.e., $F_{a_{i 1}}, F_{a_{i 2}}, \ldots, F_{a_{i r}}$ responsible for generating $r$ nodes. The resulting $r$ equations are given, in matrix form, by

$$
A z=b
$$

where 


$$
\begin{aligned}
& \boldsymbol{A}=\left[\begin{array}{cccc}
G_{i}\left(x_{n_{1}}, x_{a_{i 1}}\right) & G_{i}\left(x_{n_{1}}, x_{a_{i 2}}\right) & \cdots & G_{i}\left(x_{n_{1}}, x_{a_{i r}}\right) \\
G_{i}\left(x_{n_{2}}, x_{a_{i 1}}\right) & G_{i}\left(x_{n_{2}}, x_{a_{i 2}}\right) & \cdots & G_{i}\left(x_{n_{2}}, x_{a_{i r}}\right) \\
\vdots & \vdots & \vdots & \vdots \\
G_{i}\left(x_{n_{r}}, x_{a_{i 1}}\right) & G_{i}\left(x_{n_{r}}, x_{a_{i 2}}\right) & \cdots & G_{i}\left(x_{n_{r}}, x_{a_{i r}}\right)
\end{array}\right]_{r \times r} \\
& \boldsymbol{z}=\left[\begin{array}{llll}
F_{a_{i 1}} & F_{a_{i 2}} & \cdots & F_{a_{i r}}
\end{array}\right]_{r \times 1}^{T} \\
& \boldsymbol{b}=-\left[G_{i}\left(x_{n_{1}}, x_{i f}\right) \quad G_{i}\left(x_{n_{2}}, x_{i f}\right) \cdots \quad G_{i}\left(x_{n_{r}}, x_{i f}\right)\right]_{r \times 1}^{T}
\end{aligned}
$$

The superscript $T$ denotes vector transposition. Once again, the same procedure is repeated for every excitation force independently.

\section{Multiple Fixed Nodes}

A fixed node is a point on a beam having both zero deflection as well as zero slope. To create $r$ fixed nodes, $2 r$ control forces are required. Physically, these two forces can produce the transverse and rotational displacements required to create the fixed node. The $2 r$ equations with $2 r$ unknowns can be identified by replacing $r$ with $2 r$ in Equation (17). The first $r$ equations are derived by substituting $x=x_{n_{1}}, x_{n_{2}}, \ldots, x_{n_{r}}$ in Equation(17) and setting them equal to zero. The second $r$ equations are derived by taking the derivative of Equation (17) with respect to $x$ and then substituting $x=x_{n_{1}}, x_{n_{2}}, \ldots, x_{n_{r}}$ and setting them equal to zero. The resulting linear algebraic equations, in matrix form, is given by

$$
\boldsymbol{A z}=\boldsymbol{b}
$$

where

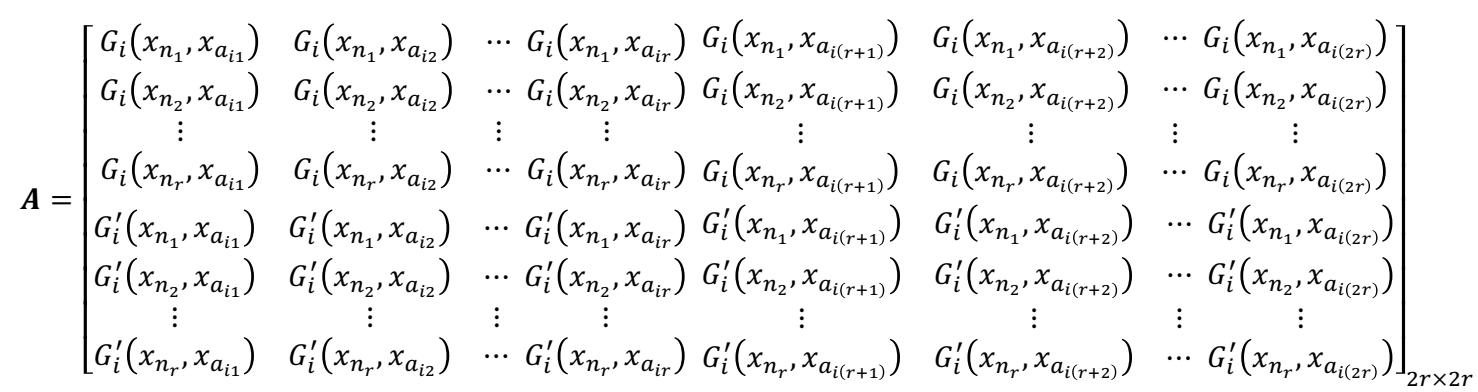

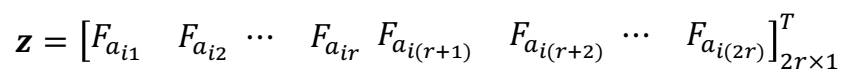

$$
\begin{aligned}
& \boldsymbol{b}=-\left[G_{i}\left(x_{n_{1}}, x_{i f}\right) \quad G_{i}\left(x_{n_{2}}, x_{i f}\right) \cdots \quad G_{i}\left(x_{n_{r}}, x_{i f}\right) G_{i}^{\prime}\left(x_{n_{1}}, x_{i f}\right) \quad G_{i}^{\prime}\left(x_{n_{2}}, x_{i f}\right) \cdots \quad G_{i}^{\prime}\left(x_{n_{r}}, x_{i f}\right)\right]_{2 r \times 1}^{T}
\end{aligned}
$$

and $G^{\prime}(x, u)$ is the derivative of the Green's function with respect to $x$. 
After calculating the amplitudes of the control forces, the steady-state deflection can be calculated using Equation (17) and then the total steady-state response is obtained using Equation (9).

\section{CONTROL FORCE REALIZATION}

The designed control force can be realized using passive elements such a spring connected between a point on the beam and ground or an oscillator containing a spring and a mass attached to the beam as seen in Figure 2. It should be noted that the required number of passive elements matches the number of applied control forces needed to impose the $\boldsymbol{r}$ nodes. At this stage, it is assumed that the applied control forces have been calculated using the numerical procedure outlined in Section 4.

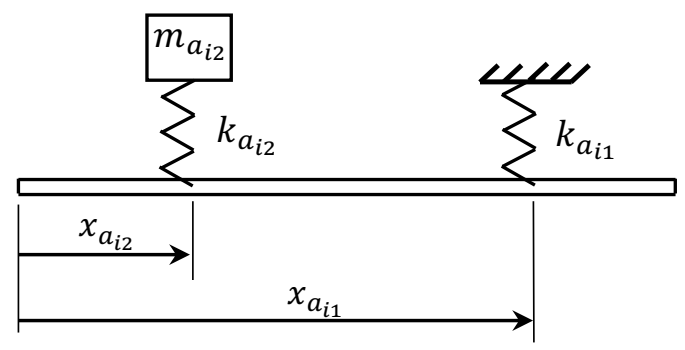

Figure 2. Configuration of a harmonically excited elastic beam with attached passive elements.

If the spring element is used, then the value of the stiffness can be calculated by equating the spring reaction force exerted on the beam with the magnitude of the applied control force:

$$
\boldsymbol{F}_{a_{i 1}}=\boldsymbol{k}_{a_{i 1}} \boldsymbol{W}\left(\boldsymbol{x}_{a_{i 1}}\right)
$$

and, therefore, the spring stiffness value can be calculated by

$$
\boldsymbol{k}_{a_{i 1}}=\frac{\boldsymbol{F}_{a_{i 1}}}{W\left(x_{a_{i 1}}\right)}
$$

where the beam deflection amplitude at $\boldsymbol{x}_{\boldsymbol{a}_{\boldsymbol{i 1}}}$ can be calculated using Equation (17) by substituting $\boldsymbol{x}=\boldsymbol{x}_{\boldsymbol{a}_{\boldsymbol{i 1}}}$. It is worth noting that when the applied control force location matches the node location (collocated case), then the denominator of Equation (34) becomes zero resulting in a value of infinity for the stiffness. Physically, this case can be achieved by rigidly attaching the beam to the ground. Alternatively, to obtain a finite value for the spring stiffness, the spring attachment location should not coincide with the node location (noncollocated case).

If an oscillator with mass and spring is attached to the beam at location $\boldsymbol{x}_{\boldsymbol{a}_{\boldsymbol{i}}}$, then the values of the mass and spring stiffness can be calculated as follows (Foda and Alsaif 2009). In this case, the equation of motion for the oscillator dynamics is given by

$$
m_{a_{i 2}} \ddot{y}(t)+k_{a_{i 2}}\left[y(t)-w\left(x_{a_{i 2}}, t\right)\right]=0
$$

where $\boldsymbol{y}(\boldsymbol{t})$ is the absolute displacement of the oscillator mass $\boldsymbol{m}_{\boldsymbol{a}_{\boldsymbol{i}}}$. Since the system is linear, the response $\boldsymbol{y}(\boldsymbol{t})$ takes the form 


$$
y(t)=Y e^{j \omega_{i} t}
$$

where $\boldsymbol{Y}$ is the amplitude of the oscillator displacement. The reaction force exerted by the oscillator on the beam is given by

$$
\boldsymbol{F}_{a_{i 2}}=\boldsymbol{k}_{a_{i 2}}\left[\boldsymbol{W}\left(\boldsymbol{x}_{a_{i 2}}\right)-\boldsymbol{Y}\right]
$$

From Equations (35), (36), and (37), it can be easily shown that the reaction force exerted by the oscillator on the beam can be written as

$$
F_{a_{i 2}}=\alpha_{a_{i 2}}\left(\omega_{i}\right) W\left(x_{a_{i 2}}\right)
$$

where $\boldsymbol{\alpha}_{\boldsymbol{a}_{\boldsymbol{i} 2}}$ is the control force gain that is given by

$$
\alpha_{a_{i 2}}\left(\omega_{i}\right)=\frac{m_{a_{i 2}} \omega_{i}^{2} k_{a_{i 2}}}{k_{a_{i 2}}-m_{a_{i 2}} \omega_{i}^{2}}=\frac{\omega_{i}^{2} k_{a_{i 2}}}{\omega_{a}^{2}-\omega_{i}^{2}}
$$

where $\boldsymbol{\omega}_{\boldsymbol{a}}$ is the natural frequency of the oscillator, in $\mathrm{rad} / \mathrm{s}$, and is given by

$$
\omega_{a}=\sqrt{\frac{k_{a_{i 2}}}{m_{a_{i 2}}}}
$$

It is to be noted that the applied control force gain in Equation (39) can be calculated after calculating the control force as well as the amplitude of the beam deflection at $\boldsymbol{x}_{\boldsymbol{a}_{i 2}}$ based on the procedures in Section 4.

When the node location $\left(\boldsymbol{x}_{\boldsymbol{n}}\right)$ coincide with the oscillator location $\left(\boldsymbol{x}_{\boldsymbol{a}_{i 2}}\right)$, then the control force gain in Equation (38) approaches infinity. In this case, the natural frequency of the oscillator becomes equal to the excitation frequency, as seen in Equation (39), and the values of oscillator mass and oscillator stiffness can be calculated from

$$
\frac{k_{a_{i 2}}}{m_{a_{i 2}}}=\omega_{i}^{2}
$$

where either the oscillator stiffness is calculated for a given value of oscillator mass or vice versa. This freedom in the selection of the oscillator parameters can be used to select the values that results in minimizing the oscillator vibrational amplitude.

For the noncollocated case, the control force gain has a finite value and Equation (39) can be used to calculate the oscillator mass for a given stiffness or vice versa.

\section{SIMULATIONS AND DISCUSSIONS}

In this section, numerical experiments are performed using the procedure outlined in the previous sections to create nodes and fixed nodes in a vibrating beam. The outlined numerical methods are applied on simply supported and cantilever beams, although the method is applicable to beams having any end supports.

It is to be noted that all the parameters and variables used in the following examples are dimensionless, which are defined in Equations (12) to (14). These parameters can be easily transformed to their dimensional counterpart 
once all the parameters of the beam structure are defined. Consequently, all the parameters stated in the following examples are shown without dimensions

\section{Collocated Case: Undamped Simply Supported Beam with One Node and Fixed Node and Two Frequencies}

First, we consider applying the numerical procedure outlined in the previous sections on a uniform simply supported beam and a uniform cantilever beam. For a uniform undamped simply supported beam, we consider the example reported by Cha and Ren (2006) of length $L=1$, excited by two concentrated harmonic forces of amplitude $F_{0}=1$, excitation frequencies $\omega_{1}=17$ and $\omega_{2}=43$, and excitation location $x_{1 f}=x_{2 f}=0.27$. It is desired to impose a node at the location $x_{n}=0.65$ using one collocated control force for each excitation frequency, i.e., $x_{a_{11}}=x_{a_{21}}=0.65$. For comparison, a fixed node is also designed for the same beam system using two control forces for each excitation frequency, one force applied at $x_{a_{i 1}}=0.65$ and the other one applied at $x_{a_{i 2}}=0.25$. The calculated applied control forces amplitudes and oscillators parameters are shown in Table 1. The resulting steady-state deflections of the beam for the uncontrolled case (red dashed line), one node case (orange dash-dotted line), and fixed node case (blue solid line) are shown in Figure 3. As seen in the figure, a node at exactly $x_{n}=0.65$ is created for both deflections resulting from the two excitation forces. Furthermore, the vibrations have been diminished over the complete beam span when a fixed node is enforced compared with the node case for both excitation frequencies. It is noted that Cha and Ren (2006) have used the assumed modes method to solve the same problem. They have used the Matlab routine $f$ solve to solve highly nonlinear simultaneous equations that requires initial guesses for the unknowns to obtain comparable results.

Table 1. Calculated control forces magnitudes and oscillators parameters.

\begin{tabular}{|c|c|c|c|c|c|}
\hline Case & $\begin{array}{c}\text { Excitation } \\
\text { Frequency }(\omega)\end{array}$ & $\begin{array}{c}\text { Oscillator } \\
\text { Location }\left(x_{a_{i}}\right)\end{array}$ & $\begin{array}{l}\text { Chosen } \\
\text { Stiffness }\end{array}$ & Mass & $\begin{array}{c}\text { Applied Control Force } \\
\text { Amplitude }\end{array}$ \\
\hline \multirow{2}{*}{ One Node } & 17 & 0.65 & 5 & $\begin{array}{l}1.73010 \\
\times 10^{-2}\end{array}$ & -1.15671 \\
\hline & 43 & 0.65 & 5 & $\begin{array}{l}2.70416 \\
\times 10^{-3}\end{array}$ & $8.94739 \times 10^{-1}$ \\
\hline \multirow{4}{*}{$\begin{array}{l}\text { One Fixed } \\
\text { Node }\end{array}$} & \multirow{2}{*}{17} & 0.65 & 5 & $\begin{array}{l}1.73010 \\
\times 10^{-2}\end{array}$ & $-1.18303 \times 10^{-2}$ \\
\hline & & 0.25 & 5 & $\begin{array}{l}1.72906 \\
\times 10^{-2}\end{array}$ & -1.03984 \\
\hline & \multirow{2}{*}{43} & 0.65 & 5 & $\begin{array}{l}2.70416 \\
\times 10^{-3}\end{array}$ & $-1.10415 \times 10^{-2}$ \\
\hline & & 0.25 & 5 & $\begin{array}{l}2.70249 \\
\times 10^{-3}\end{array}$ & $-9.92397 \times 10^{-1}$ \\
\hline
\end{tabular}



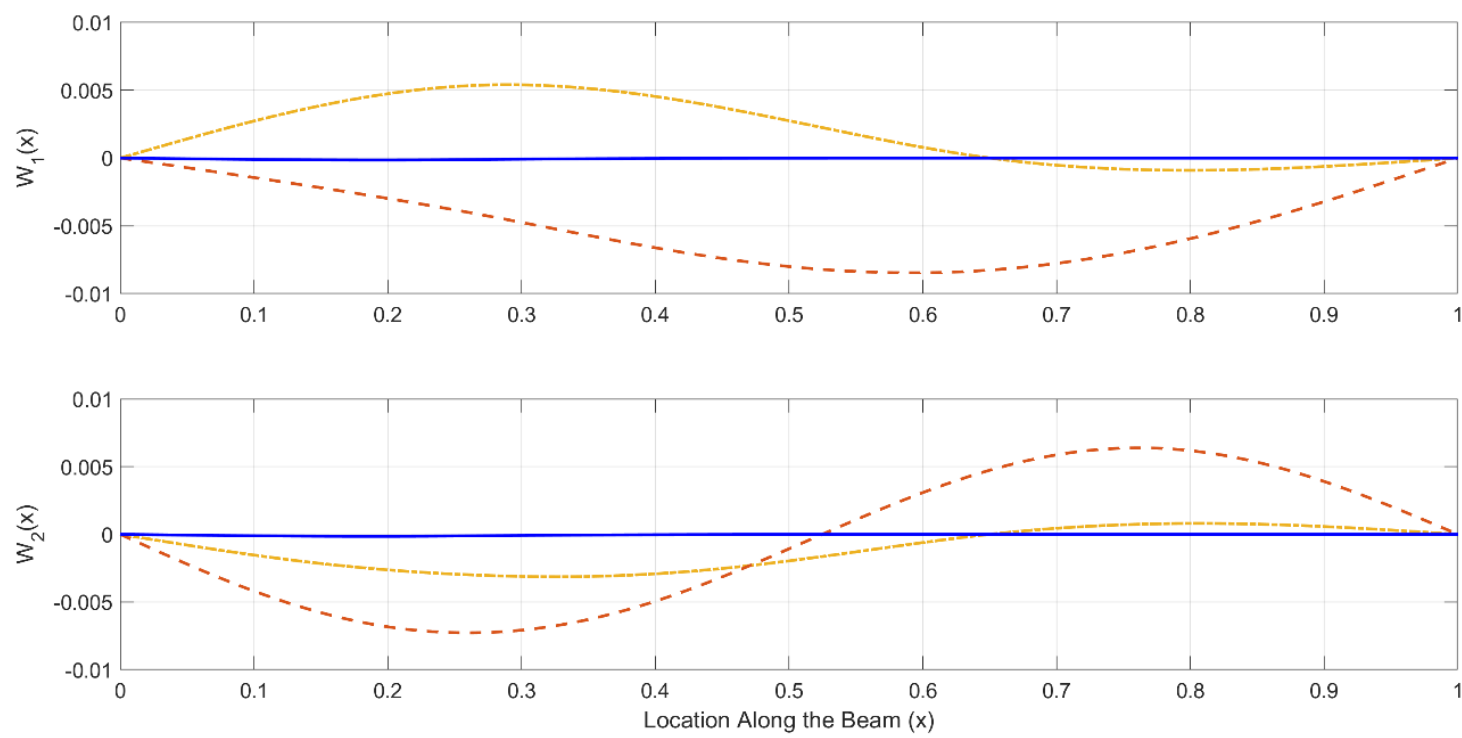

Figure 3. The steady state deflection for the uncontrolled case (red dashed line), one node case (orange dashdotted line), and fixed node case (blue solid line) of an undamped simply supported beam when $\omega_{1}=17, \omega_{2}=43$, $\boldsymbol{x}_{\boldsymbol{i f}}=0.27, \boldsymbol{x}_{\boldsymbol{n}}=0.65, \boldsymbol{x}_{\boldsymbol{a}_{11}}=\boldsymbol{x}_{\boldsymbol{a}_{\mathbf{2}}}=\mathbf{0 . 6 5}$ for the node case, and $\boldsymbol{x}_{\boldsymbol{a}_{\boldsymbol{i 1}}}=\mathbf{0 . 6 5}$ and $\boldsymbol{x}_{\boldsymbol{a}_{\boldsymbol{i} 2}}=\mathbf{0 . 2 5}$ for the fixed node case.

\section{Collocated Case: Undamped Cantilever Beam with One Node and Fixed Node and Two Frequencies}

Next, it is desired to apply the outlined numerical procedure on a cantilever beam. This example is reported by Cha and Ren (2006), in which, an undamped beam is subjected to excitation forces at $x_{1 f}=x_{2 f}=0.85$ with two frequencies $\omega_{1}=12$ and $\omega_{2}=31$. A node and fixed node is desired at $x_{n}=1$ using two collocated control forces for the node case (i.e., $x_{a_{11}}=x_{a_{21}}=1$ ) and four control forces applied at $x_{a_{i 1}}=1$ and $x_{a_{i 2}}=0.84$ for the fixed node case. The calculated applied control forces amplitudes and oscillators parameters are shown in Table 2. The resulting steady-state deflections of the beam for the uncontrolled case (red dashed line), one node case (orange dash-dotted line), and fixed node case (blue solid line) are shown in Figure 4. As seen in this figure, a node is created at the tip of the beam without using any rigid support. In addition, enforcing a zero slope at the node location has reduced the vibration amplitudes over the total beam length.

Table 2. Calculated control forces magnitudes and oscillators parameters.

\begin{tabular}{|c|c|c|c|c|c|}
\hline Case & $\begin{array}{c}\text { Excitation } \\
\text { Frequency }(\boldsymbol{\omega})\end{array}$ & $\begin{array}{c}\text { Oscillator } \\
\text { Location }\left(\boldsymbol{x}_{\boldsymbol{a}_{\boldsymbol{i}}}\right)\end{array}$ & $\begin{array}{c}\text { Chosen } \\
\text { Stiffness }\end{array}$ & Mass & $\begin{array}{c}\text { Applied Control Force } \\
\text { Amplitude }\end{array}$ \\
\hline \multirow{3}{*}{ One Node } & 12 & 1 & 7 & $\begin{array}{l}4.86111 \\
\times 10^{-2}\end{array}$ & -1.23135 \\
\cline { 2 - 6 } & 31 & 1 & 7 & $\begin{array}{l}7.28408 \\
\times 10^{-3}\end{array}$ & $-5.77891 \times 10^{-1}$ \\
\hline
\end{tabular}




\begin{tabular}{|c|c|c|c|c|c|}
\hline & \multirow{2}{*}{12} & 1 & 7 & $\begin{array}{l}4.86111 \\
\times 10^{-2}\end{array}$ & $-4.83192 \times 10^{-2}$ \\
\cline { 3 - 6 } $\begin{array}{c}\text { One Fixed } \\
\text { Node }\end{array}$ & \multirow{2}{*}{31} & 0.84 & 7 & $\begin{array}{l}4.85994 \\
\times 10^{-2}\end{array}$ & $-9.52000 \times 10^{-1}$ \\
\cline { 2 - 6 } & 1 & 7 & $\begin{array}{l}7.28408 \\
\times 10^{-3}\end{array}$ & $3.28769 \times 10^{-3}$ \\
\cline { 3 - 6 } & 0.84 & 7 & $\begin{array}{l}7.28419 \\
\times 10^{-3}\end{array}$ & -1.06413 \\
\hline
\end{tabular}
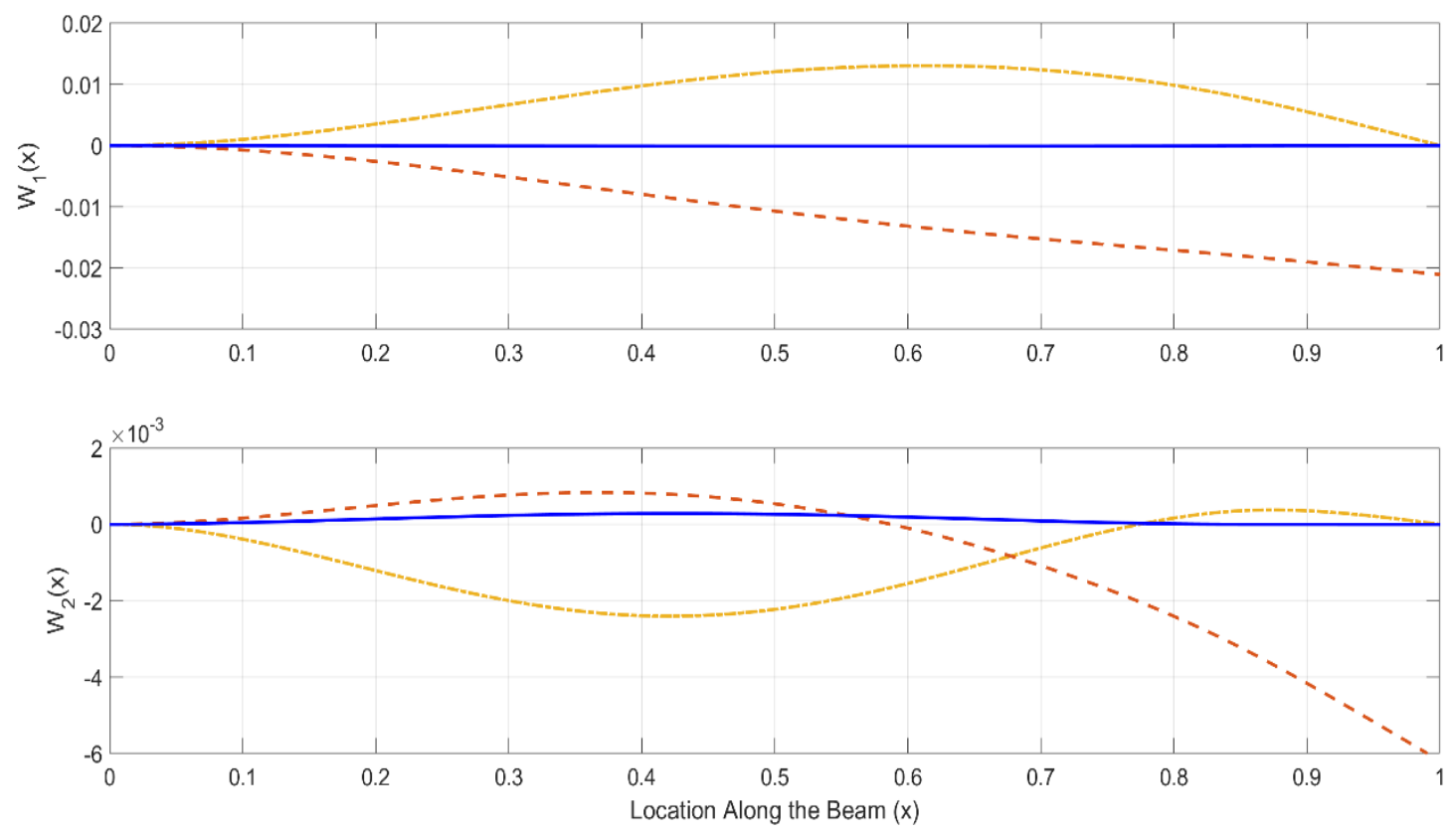

Figure. 4. The steady state deflection for the uncontrolled case (red dashed line), one node case (orange dashdotted line), and fixed node case (blue solid line) of an undamped cantilever beam when $\omega_{1}=12, \omega_{2}=31$, $\boldsymbol{x}_{\boldsymbol{i f}}=0.85, \boldsymbol{x}_{\boldsymbol{n}}=1, \boldsymbol{x}_{\boldsymbol{a}_{11}}=\boldsymbol{x}_{\boldsymbol{a}_{21}}=\mathbf{1}$ for the node case, and $\boldsymbol{x}_{\boldsymbol{a}_{\boldsymbol{i 1}}}=\mathbf{1}$ and $\boldsymbol{x}_{\boldsymbol{a}_{\boldsymbol{i 2}}}=\mathbf{0 . 8 4}$ for the fixed node case.

\section{Noncollocated Case: Damped Simply Supported Beam with Fixed Node and Two Frequencies}

Figure 5 depicts the steady-state deflections for a damped simply supported beam subjected to two harmonic force excitations with frequencies $\omega_{1}=50$ and $\omega_{2}=100$ that act at $x_{i f}=0.8$. The damping coefficients are $c_{1}=0.1$ and $c_{2}=0.001$. Based on a certain application, it is desired to apply the outlined numerical procedure to impose a fixed node at $x_{n}=0.3$ using two control forces acting at $x_{a_{i 1}}=0.6$ and $x_{a_{i 2}}=0.75$. The calculated applied control forces amplitudes and oscillators parameters are shown in Table 3 . The resulting steady-state deflections of the beam for the uncontrolled case (red dashed line) and fixed node case (blue solid line) are shown in Figure 4. Not only zero deflection and zero slope is achieved at the node location for both excitation forces but also the beam vibrations are diminished in the range $0 \leq x \leq 0.7$. 
Table 3. Calculated control forces magnitudes and oscillators parameters.

\begin{tabular}{|c|c|c|c|c|c|}
\hline \multirow{2}{*}{ Case } & $\begin{array}{c}\text { Excitation } \\
\text { Frequency }(\boldsymbol{\omega})\end{array}$ & $\begin{array}{c}\text { Oscillator } \\
\text { Location }\left(\boldsymbol{x}_{\boldsymbol{a}_{\boldsymbol{i}}}\right)\end{array}$ & $\begin{array}{c}\text { Chosen } \\
\text { Stiffness }\end{array}$ & Mass & $\begin{array}{c}\text { Applied Control Force } \\
\text { Amplitude }\end{array}$ \\
\hline \multirow{2}{*}{$\begin{array}{c}\text { One Fixed } \\
\text { Node }\end{array}$} & 50 & 0.6 & 5 & $2 \times 10^{-3}$ & $1.23247 \times 10^{-1}$ \\
\cline { 2 - 6 } & 100 & 0.75 & 5 & $\begin{array}{l}2.00066 \\
\times 10^{-3}\end{array}$ & -1.04578 \\
\cline { 2 - 6 } & & 0.6 & 5 & $5 \times 10^{-4}$ & $1.582788 \times 10^{-1}$ \\
\cline { 2 - 5 } & & 0.75 & 5 & $\begin{array}{l}5.00170 \\
\times 10^{-4}\end{array}$ & -1.31056 \\
\hline
\end{tabular}
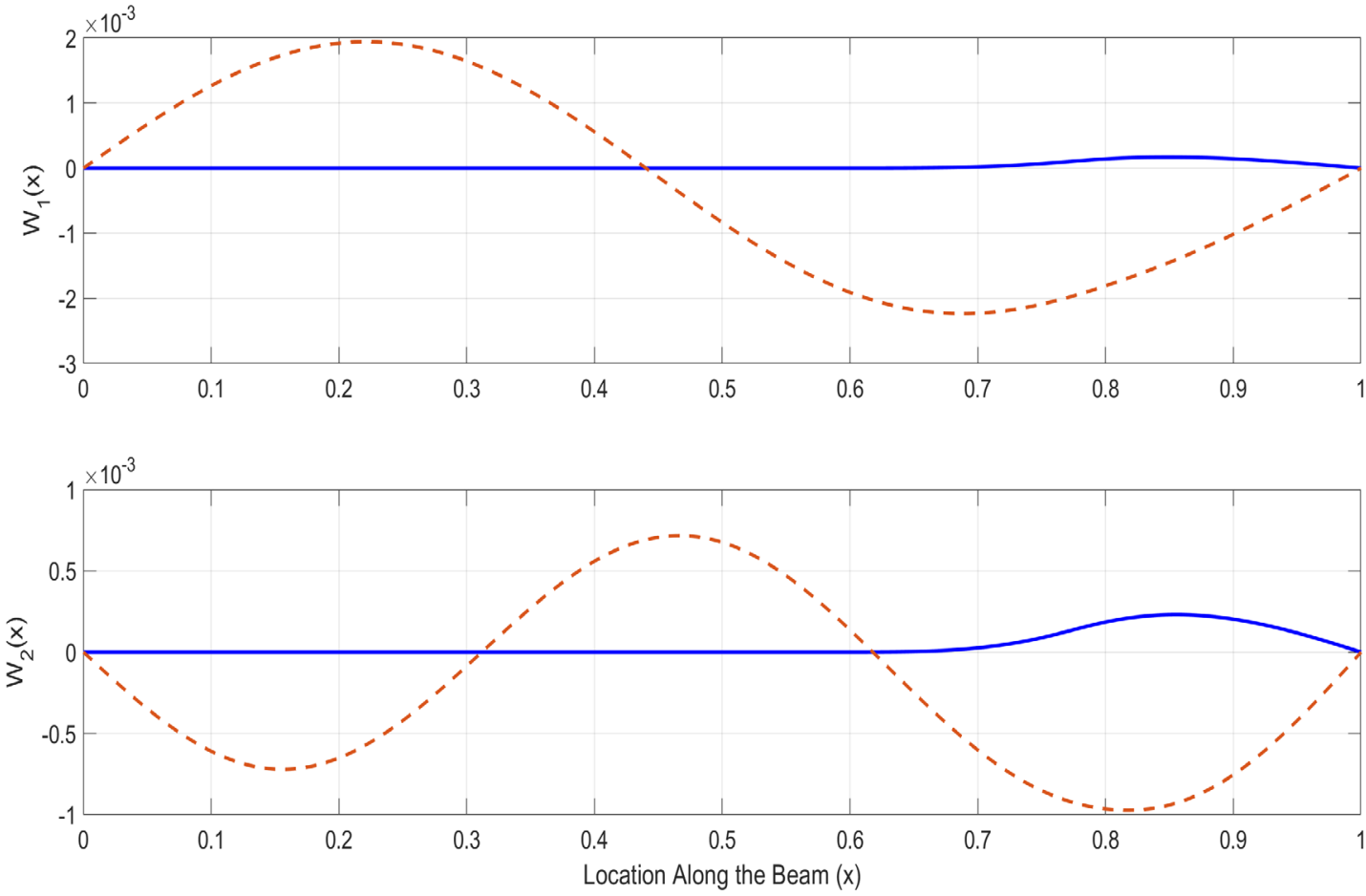

Figure 5. The steady state deflections for the uncontrolled case (red dashed line) and fixed node case (blue solid line) of a damped simply supported beam when $\omega_{1}=12, \omega_{2}=31, \mathrm{c}_{1}=0.1, \mathrm{c}_{2}=0.001, \boldsymbol{x}_{\boldsymbol{i f}}=0.8, \boldsymbol{x}_{\boldsymbol{n}}=0.3, \boldsymbol{x}_{\boldsymbol{a}_{\boldsymbol{i 1}}}=\mathbf{0 . 6}$ and $\boldsymbol{x}_{\boldsymbol{a}_{i 2}}=\mathbf{0 . 7 5}$ 


\section{Noncollocated Case: Undamped Simply Supported Beam With Two Nodes and Two Frequencies}

Consider the example reported by Cha and Buyco (2015) for an undamped simply supported beam excited by two forces applied at $x_{1 f}=x_{2 f}=0.4$ having frequencies $\omega_{1}=80$ and $\omega_{2}=125$. It is desired to impose two nodes on the beam at locations $x_{n_{1}}=0.1$ and $x_{n_{2}}=0.6$ using two control forces for each excitation frequency applied at $x_{a_{i 1}}=0.3$ and $x_{a_{i 2}}=0.45$. The calculated applied control forces amplitudes and oscillators parameters are shown in Table 4. The resulting steady-state deflections of the beam for the uncontrolled case (red dashed line) and two nodes case (blue solid line) are shown in Figure 6. As seen in this figure, the two nodes are created at exactly the desired location. In addition, the vibrational amplitudes have been weakened along the complete beam span.

Table 4. Calculated control forces magnitudes and oscillators parameters.

\begin{tabular}{|c|c|c|c|c|c|}
\hline Case & $\begin{array}{c}\text { Excitation } \\
\text { Frequency }(\omega)\end{array}$ & $\begin{array}{c}\text { Oscillator } \\
\text { Location }\left(x_{a_{i}}\right)\end{array}$ & $\begin{array}{l}\text { Chosen } \\
\text { Stiffness }\end{array}$ & Mass & $\begin{array}{c}\text { Applied Control Force } \\
\text { Amplitude }\end{array}$ \\
\hline \multirow{4}{*}{$\begin{array}{r}\text { Two } \\
\text { Nodes }\end{array}$} & \multirow{2}{*}{80} & 0.3 & 35.57 & $\begin{array}{l}5.58552 \\
\times 10^{-3} \\
\end{array}$ & $-3.55539 \times 10^{-1}$ \\
\hline & & 0.45 & 79.56 & $\begin{array}{l}1.24742 \\
\times 10^{-2} \\
\end{array}$ & $-7.96264 \times 10^{-1}$ \\
\hline & \multirow{2}{*}{125} & 0.3 & 50.37 & $\begin{array}{l}3.24393 \\
\times 10^{-3}\end{array}$ & $-5.10711 \times 10^{-1}$ \\
\hline & & 0.45 & 85.51 & $\begin{array}{l}5.50980 \\
\times 10^{-3}\end{array}$ & $-8.70156 \times 10^{-1}$ \\
\hline
\end{tabular}
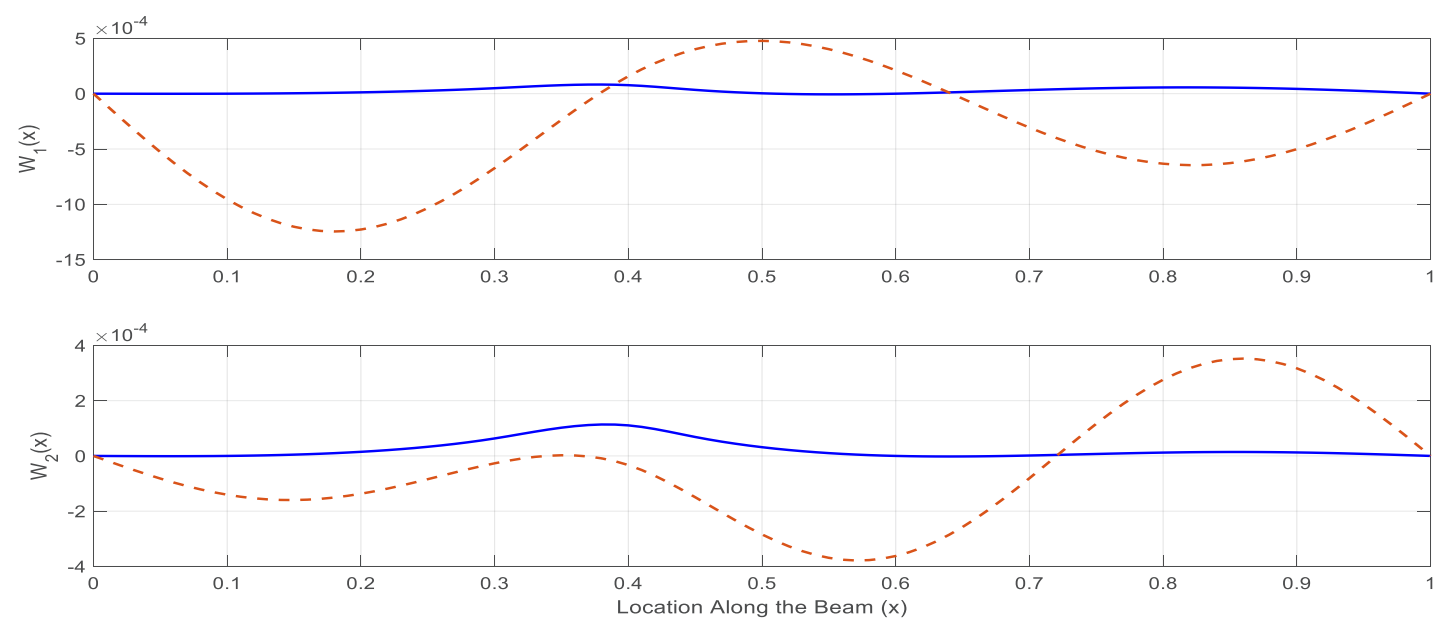

Figure. 6. The steady state deflections for the uncontrolled case (red dashed line) and two nodes case (blue solid line) of an undamped simply supported beam when $\omega_{1}=80, \omega_{2}=125, \boldsymbol{x}_{\boldsymbol{i f}}=0.4$,

$$
\boldsymbol{x}_{\boldsymbol{n}_{1}}=0.1, \boldsymbol{x}_{\boldsymbol{n}_{2}}=0.6, \boldsymbol{x}_{\boldsymbol{a}_{\boldsymbol{i 1}}}=0.3 \text {, and } \boldsymbol{x}_{\boldsymbol{a}_{\boldsymbol{i}}}=0.45 \text {. }
$$




\section{Noncollocated Case: Undamped Cantilever Beam with Three Nodes and Three Frequencies}

Consider the example reported by Cha and Buyco (2015) for an undamped cantilever beam excited by three forces applied at $x_{1 f}=x_{2 f}=x_{3 f}=0.23$ having frequencies $\omega_{1}=140, \omega_{2}=185$, and $\omega_{3}=230$. It is required to impose three nodes on the beam at locations $x_{n 1}=0.05, x_{n_{2}}=0.5$ and $x_{n 3}=0.8$ using three control forces for each excitation frequency applied at $x_{a_{i 1}}=0.2, x_{a_{i 2}}=0.25$, and $x_{a_{i 3}}=0.3$. The calculated applied control forces amplitudes and oscillators parameters are shown in Table 5. The resulting steady-state deflections of the beam for the uncontrolled case (red dashed line) and three nodes case (blue solid line) are shown in Figure 7. As seen in this figure, the vibrations have been cancelled not only at the three node locations but rather on the complete length of the beam.

Table 5. Calculated control forces magnitudes and oscillators parameters.

\begin{tabular}{|c|c|c|c|c|c|}
\hline Case & 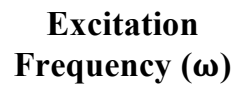 & $\begin{array}{c}\text { Oscillator } \\
\text { Location }\left(x_{a_{i}}\right)\end{array}$ & $\begin{array}{l}\text { Chosen } \\
\text { Stiffness }\end{array}$ & Mass & $\begin{array}{c}\text { Applied Control Force } \\
\text { Amplitude }\end{array}$ \\
\hline \multirow{9}{*}{$\begin{array}{l}\text { Three } \\
\text { Nodes }\end{array}$} & \multirow{3}{*}{140} & 0.20 & 34.74 & $\begin{array}{l}1.77309 \\
\times 10^{-3}\end{array}$ & $-3.33349 \times 10^{-1}$ \\
\hline & & 0.25 & 74.21 & $\begin{array}{l}3.78691 \\
\times 10^{-3}\end{array}$ & $-7.66362 \times 10^{-1}$ \\
\hline & & 0.30 & 7.297 & $\begin{array}{l}3.72296 \\
\times 10^{-4}\end{array}$ & $8.93060 \times 10^{-2}$ \\
\hline & \multirow{3}{*}{185} & 0.20 & 36.01 & $\begin{array}{l}1.05254 \\
\times 10^{-3}\end{array}$ & $-3.38278 \times 10^{-1}$ \\
\hline & & 0.25 & 73.10 & $\begin{array}{l}2.13624 \\
\times 10^{-3}\end{array}$ & $-7.65888 \times 10^{-1}$ \\
\hline & & 0.30 & 6.477 & $\begin{array}{l}1.89248 \\
\times 10^{-4}\end{array}$ & $8.87965 \times 10^{-2}$ \\
\hline & \multirow{3}{*}{230} & 0.20 & 36.50 & $\begin{array}{l}6.90225 \\
\times 10^{-4}\end{array}$ & $-3.44292 \times 10^{-1}$ \\
\hline & & 0.25 & 73.36 & $\begin{array}{l}1.38701 \\
\times 10^{-3}\end{array}$ & $-7.65418 \times 10^{-1}$ \\
\hline & & 0.30 & 6.512 & $\begin{array}{l}1.23100 \\
\times 10^{-4}\end{array}$ & $8.81902 \times 10^{-2}$ \\
\hline
\end{tabular}



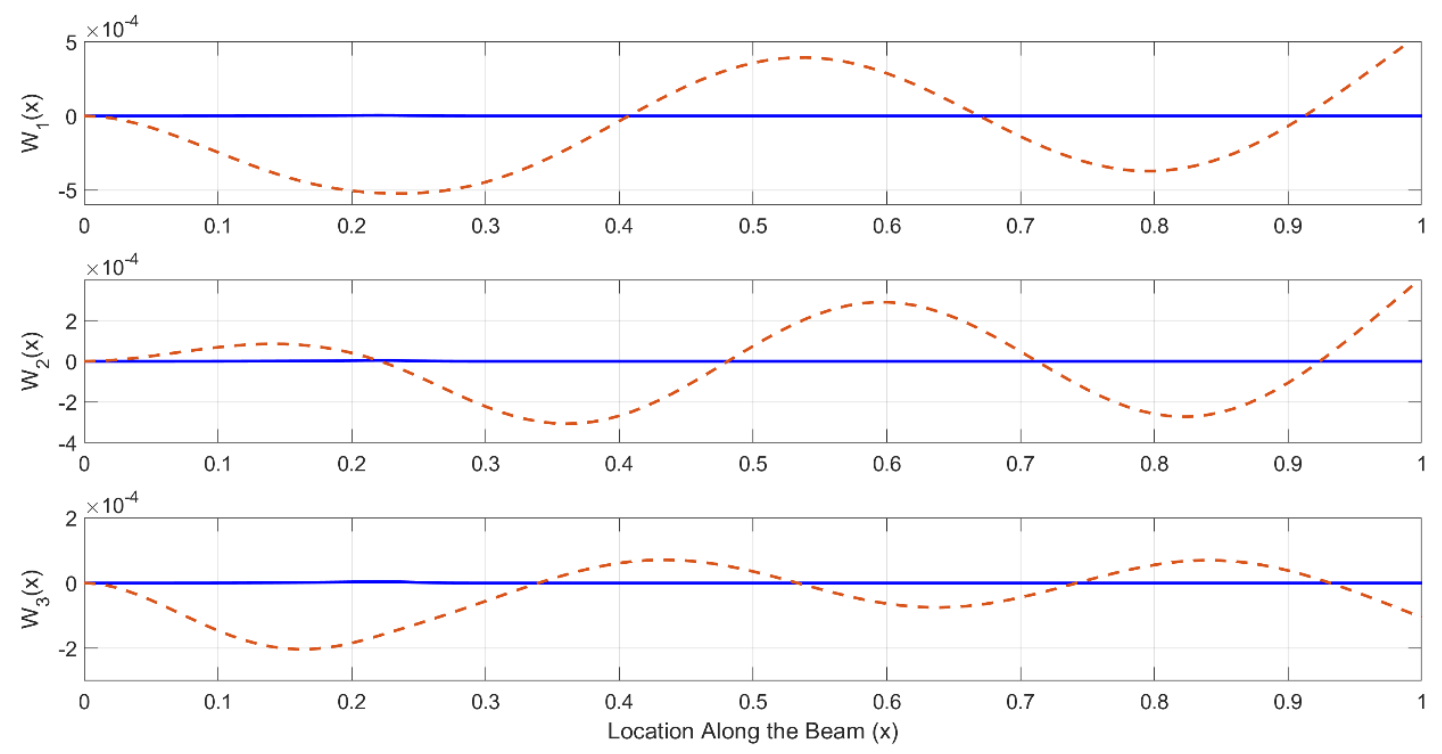

Figure 7. The steady state deflections for the uncontrolled case (red dashed line) and three nodes case (blue solid line) of an undamped cantilever beam when $\omega_{1}=140, \omega_{2}=185, \omega_{3}=230, x_{i f}=0.23, x_{n_{1}}=0.05, x_{n_{2}}=0.5$,

$$
\begin{gathered}
\boldsymbol{x}_{\boldsymbol{n}_{3}}=0.8, \boldsymbol{x}_{\boldsymbol{a}_{\boldsymbol{i}}}=0.2, \boldsymbol{x}_{\boldsymbol{a}_{\boldsymbol{i} 2}}=0.25, \\
\text { and } \boldsymbol{x}_{\boldsymbol{a}_{\boldsymbol{i}}}=0.3 \text {. }
\end{gathered}
$$

\section{CONCLUSION}

In this paper, Green's function is utilized to develop an exact analytical solution for the control forces required to impose points with zero deflection, called a node, and zero slope, called a fixed node, on a beam structure excited by disturbances having multiple frequencies. The designed control forces can be realized using oscillators having passive elements, such as springs and masses, attached to the beam. The principle of superposition allows the numerical procedure to be applied for each excitation force independently, which greatly simplifies the computations for the designed control forces. In general, each node requires a control force to be applied at specified location on a beam, whereas a fixed node requires two control forces. The resulting equations for the control forces are linear algebraic equations, which can be solved using Gauss elimination. The oscillators' parameters can be calculated after calculating the required control forces magnitudes with a freedom to properly select the value of one parameter and then compute the other one. This freedom in selecting the oscillators parameters can be used to choose the parameters that minimizes a certain objective function. The numerical procedure is suitable to perform parametric study of any one of the beam structure parameters, such as the oscillator location, oscillator mass, or oscillator spring to investigate its effect on the beam deflection. The validity of the numerical technique is demonstrated by several numerical examples with different cases, which proves that the method can control and reduce the vibrations not only at the desired points, but also along regions, and may be the complete beam span. 


\section{REFERENCES}

Albassam, B.A. 2019. Creating nodes at selected locations in a harmonically excited structure using feedback control and green's function. Shock and Vibration 2019, Article ID 3261628. https://doi.org/10.1155/2019/3261628.

Alsaif, K. \& Foda, M.A. 2002. Vibration suppression of a beam structure by intermediate masses and springs. Journal of Sound and Vibration 256: 629-645.

Banerjee, J.R. \& Sobey, A.J. 2004. Further investigation into eigenfrequencies of a two-part beam-mass system. Journal of Sound and Vibration 265: 899-908.

Cha, P.D. 2001. Natural frequencies of a linear elastica carrying any number of sprung masses. Journal of Sound and Vibration 247: 185-194.

Cha, P.D. 2005. Enforcing nodes at required locations in a harmonically excited structure using simple oscillators. Journal of Sound and Vibration 279: 799-816.

Cha, P.D. \& Buyco, K. 2015. An efficient method for tuning oscillator parameters in order to impose nodes on a linear structure excited by multiple harmonics. Journal of Vibration and Acoustics 137.

Cha, P.D. \& Pierre, C. 1999. Imposing nodes to the normal modes of a linear elastic structure. Journal of Sound and Vibration 219: 669-687.

Cha, P.D. \& Ren, G. 2006. Inverse problem of imposing node to suppress vibration for a structure subjected to multiple harmonic excitations. Journal of Sound and Vibration 290: 425-447.

Cha, P.D. \& Zhou, X. 2006. Imposing points of zero displacements and zero slopes along any linear structure during harmonic excitations. Journal of Sound and Vibration 297: 55-71.

Foda, M.A. \& Albassam, B.A. 2006. Vibration confinement in a general beam structure during harmonic excitation. Journal of Sound and Vibration 295: 491-517.

Foda, M.A. \& Alsaif, K. 2009. Control of lateral vibrations and slopes at desired locations along vibrating beams. Journal of Vibration and Control 15: 1649-1678.

Frham, H. 1911. Device for damping vibrations of Bodies. US Patent No. 989958.

Ginsberg, J.H. 2001. Mechanical and Structural Vibrations: Theory and Applications. John Wiley and Sons, New York.

Korenev, B.G. \& Reznikov, L.M. 1993. Dynamic Vibration Absorbers: Theory and Technical Applications. John Wiley, New York.

Low, K.H. 2003. Frequencies of beam carrying multiple masses: Rayleigh estimation versus eigenanalysis solutions. Journal of Sound and Vibration 268: 843-853.

Ormondroyd, J. \& Den Hartog, J.P. 1928. The theory of dynamic vibration absorbers. Transactions of the ASME 50: 9-22.

Ram, Y.M. 2002. Nodal control of a vibrating rod. Mechanical Systems and Signal Processing 16: 69-81.

Roach, G. F. 1970. Green's Functions: Introductory Theory with Applications. Van Nostrand Reinhold Company, London.

Roach, G. F. 1982. Greens Functions. Cambridge University Press, Cambridge.

Singh, A.N. \& Ram, Y.M. 2003. Dynamic absorption in a vibrating beam. Proceedings of the Institution of Mechanical Engineers: 187-197.

Wu, J.S. \& Chou, H.M. 1999. A new approach for determining the natural frequencies and mode shapes of a uniform beam carrying any number of sprung masses. Journal of Sound and Vibration 220: 451-468. 